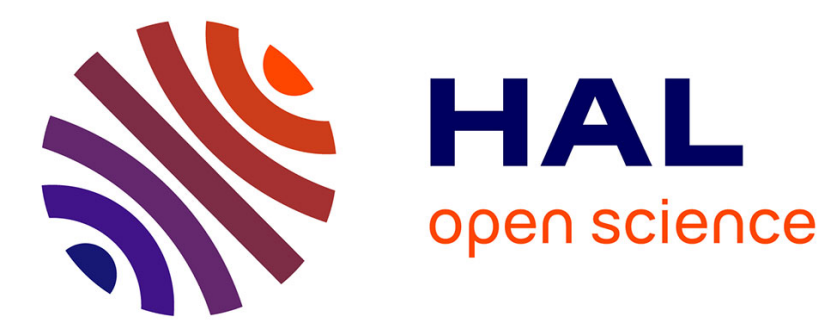

\title{
Breakdown of adiabatic invariance of fast ions in spherical tokamaks
}

Dominique F Escande, F Sattin

\section{To cite this version:}

Dominique F Escande, F Sattin. Breakdown of adiabatic invariance of fast ions in spherical tokamaks. Nuclear Fusion, 2021. hal-03266629v2

\section{HAL Id: hal-03266629 \\ https://hal.science/hal-03266629v2}

Submitted on 30 Jun 2021 (v2), last revised 11 May 2022 (v3)

HAL is a multi-disciplinary open access archive for the deposit and dissemination of scientific research documents, whether they are published or not. The documents may come from teaching and research institutions in France or abroad, or from public or private research centers.
L'archive ouverte pluridisciplinaire HAL, est destinée au dépôt et à la diffusion de documents scientifiques de niveau recherche, publiés ou non, émanant des établissements d'enseignement et de recherche français ou étrangers, des laboratoires publics ou privés. 


\title{
Breakdown of adiabatic invariance of fast ions in spherical tokamaks
}

\author{
D.F. Escande ${ }^{1}$, F. Sattin ${ }^{2}$ \\ ${ }^{1}$ Aix-Marseille Université, CNRS, PIIM, UMR 7345, Marseille, France \\ ${ }^{2}$ Consorzio RFX (CNR, ENEA, INFN, Università di Padova, Acciaierie Venete Spa), \\ Padova, Italy
}

\begin{abstract}
.
The dynamics of fast particles corresponding to $\mathcal{O}(100) \mathrm{keV}$ in NSTX, or of alpha particles in a properly rescaled reactor-grade spherical tokamak, is computed numerically through integration of the full equations of motion. The magnetic moment $\mu$ of these particles has large oscillations, and even chaotic ones, in a sizable domain of the machine. This has both practical and physical consequences. First, when $\mu$ has large oscillations, the use of guiding-center or gyrokinetic calculations for such orbits is questionable. Second, the capability of these particle to excite Alfvénic instabilities decreases, since the velocity of the particle fluctuates with respect to the phase-velocity of the Alfvén wave, which imposes a fluctuating sign to the energy exchanges with this wave. Even when chaotic orbits are present, the conservation of the toroidal momentum sets strong constraints about the volume available to the particles, and their radial diffusion stays bounded.
\end{abstract}

Submitted to: Nuclear Fusion 


\section{Introduction}

Adiabatic invariants are fundamental tools in magnetized plasma theory. The conservation of the magnetic moment under slow variations of plasma parameters is a basic assumption of guiding-center theory and lies at the heart of gyrokinetic codes, which are presently the state-of-the-art instruments for the study of plasma microinstabilities.

The existence of a sufficient number of constants of motion-including the adiabatic ones-ensures regularity of the motion; conversely, the lack thereof may-although not necessarily-imply sizeable chaotic motion. Adiabatic invariance is based upon the postulate of a wide separation between the particle Larmor radius $\rho$ and cyclotron frequency $\Omega$ from the one side, and the corresponding length and frequency scales $L, \omega$ of the ambient magnetic field. In strongly magnetized laboratory plasmas this postulate is generally verified by a large margin. Yet, since $\rho, \Omega, L, \omega$ are all dependent on local conditions, it may happen that, at selected locations in the particle phase space, the scale separation fails with potential consequences upon the conservation of the adiabatic invariants. Fast particles (beam ions or alpha particles) in spherical tokamaks (ST's) combine large Larmor radii and wide variations of the magnetic fields (small $L$ 's), and thus might experience breakdown of adiabaticity under realistic conditions. A few studies dedicated to this topic confirmed this feeling [1, 2], and [3] showed that a part of fast particle orbits in MAST cannot be described by guiding-center theory. Section 3.1.1 of the review paper [4] states "the numerical study of the nonadiabaticity diffusion effect of fusion products seems to be very important for reactor scale ST".

This paper investigates the nature of the dynamics of fast (beam-like) ions in a spherical tokamak, with an axis-symmetric NSTX geometry taken as a reference, or for alpha particles in a reactor-grade spherical tokamak with appropriately rescaled size and magnetic field. This is done through numerical integration of the collisionless complete equations of motion, not the guiding-center ones. The purpose of the paper is manifold: first, we wish to extend studies $[1,2]$ by examining a much larger set of trajectories than presented in those papers. Second, we are interested in the distribution of the dynamics in adiabatic, superadiabatic and non-adiabatic. While genuine non-adiabatic dynamics may lead to chaotic motion and possibly to a strong increase of ion losses to the walls, by superadiabatic dynamics one refers to cases where the magnetic moment $\mu$ oscillates over time scales longer than the Larmor one around a constant mean value. As long as the time-averaged $\mu$ remains constant, dynamics is still regular in an axis-symmetric device; yet, finite excursions in $\mu$ may nonetheless produce some effects: for instance, increased pitch-angle diffusion due to Coulomb scattering and a decreased excitation of Alfvénic waves. It also invalidates guiding-center and gyrokinetic descriptions. Even remaining in the collisionless regime, we will show that the boundary between superadiabatic and non-adiabatic motion is fuzzy: over very long times if compared to the bounce time, but still below the experimental collision frequency, a transition from a seemingly former one to the latter may take place. 


\section{Mathematical tools}

We use a magnetic geometry corresponding to an axis-symmetric NSTX spherical tokamak, implementing the recipe suggested by Yavorskij et al [5]. The $(R, Z, \phi)$ set of cylindrical coordinates is related to the $r$ flux surface radius and $\chi$ poloidal angle through $(29,38,39)$ in [5] (see also its figure 1 and related discussion):

$$
\begin{aligned}
& R=R_{c}+\Delta(r)+r \cos (\chi) \\
& Z=k r \sin (\chi)(1-\Lambda(r) \cos (\chi))^{\alpha}
\end{aligned}
$$

In (2), the Shafranov shift $\Delta$, the elongation $k$, the triangularity $\Lambda$ have been taken by the curves published in figure (5) of [5], corresponding to the NSTX case with $\beta=23 \%$. The other parameters are $R_{c}=1.25 a$ ( $a=$ minor radius), $\alpha=1$ [6]. No up-down asymmetry was included. The magnetic fields are defined as in (3-7) of [5]). Their definition involves the safety factor $q(r)$, the poloidal current $J(r)$ and the toroidal current $I(r)$, which have been taken from the curves plotted in figures $(13,15)$ of [5]. A problem of self-consistency arises, since we use in the calculation of magnetic fields the

quantities $I, J, q$ as given known functions, whereas their argument is the flux coordinates $r$, which should be self-consistently related to the magnetic field themselves. There is no warranty, therefore, that the computed fields are truly magnetic fields, i.e., divergencefree. In order to cure this defect, we clean the fields, by removing a quantity

$$
\mathbf{B} \rightarrow \mathbf{B}-\nabla \sigma
$$

with $\sigma$ defined by Poisson equation

$$
\nabla^{2} \sigma=\nabla \cdot \mathbf{B}
$$

The correction, which turns out to be fairly small, ensures $\mathbf{B}$ to be a valid magnetic field.

Finally, we write the magnetic potential $\mathbf{A}=\left\{0, A_{Z}(R, Z), 0, A_{\phi}(R, Z)\right\}$; its components are defined as

$$
\begin{aligned}
& A_{Z}=\int^{R} d R^{\prime} B_{\phi}\left(R^{\prime}, Z\right) \\
& \frac{\partial^{2} A_{\phi}}{\partial R^{2}}+\frac{\partial^{2} A_{\phi}}{\partial Z^{2}}=-(\nabla \times \mathbf{B})_{\phi}
\end{aligned}
$$

The latter equation is solved numerically. Figure (1) displays the contour plot of the local Larmor period $2 \pi / B$ : besides being straightforwardly related to $B$, plotting this quantity is useful since it yields a visual insight of the characteristic time scale involved, which will be useful in the following.

Particle trajectories are computed from the canonical equations of motion with Hamiltonian

$$
H=\frac{P_{R}^{2}}{2}+\frac{\left(P_{Z}-A_{Z}\right)^{2}}{2}+\frac{\left(P_{\phi}-A_{\phi}\right)^{2}}{2} .
$$

Normalized units are used: particle charge and mass are taken unitary; length is normalized to the torus minor radius $a$, and the magnetic field to a reference field 


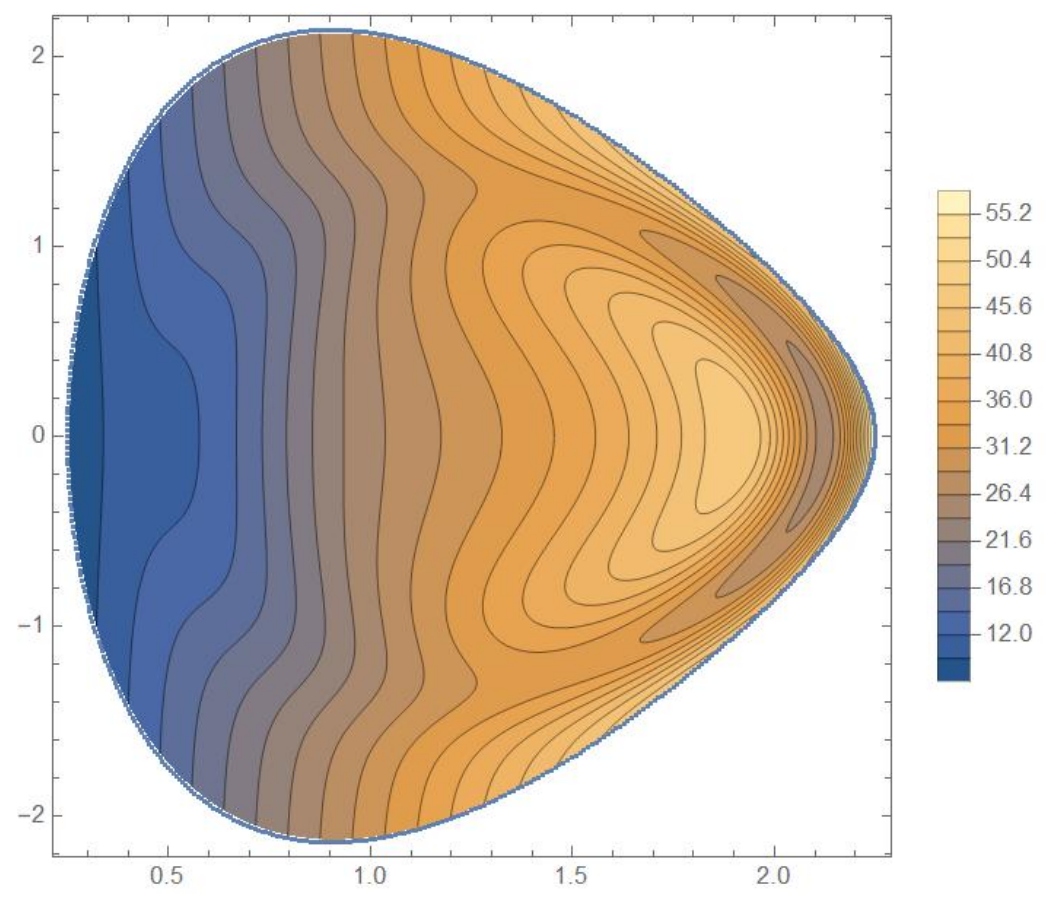

Figure 1. Contour plot of the local Larmor period $2 \pi / B$.

$B_{0}$. In order to convert from normalized units, it is convenient to use Larmor radius as a gauge: with values $a=0.7 \mathrm{~m}, B_{0}=0.3 \mathrm{~T}$, a $100-\mathrm{keV}$ deuteron with a velocity perpendicular to the magnetic field has a normalized Larmor radius $\rho / a \approx 0.2$.

In order to compute particle trajectories reliably, we have implemented a symplectic partitioned Runge-Kutta algorithm of sixth order, as described in [7]. The time step was always checked to stay well below 1/1000 of Larmor period, energy was conserved in all simulations with a relative precision not worse than $10^{-6}$ and usually better by orders of magnitude.

Since the magnetic topology is held fixed, the only control knobs in our simulations are the particles' initial conditions. There are five of them, since the angle $\phi$ is an ignorable coordinate. In the following simulations we will check the effect of varying them all, but following a few guidelines. We use $R$ as the main control parameter among the spatial coordinates; all particles are initialized on the equatorial plane $Z=0$. Concerning the velocity degrees of freedom, we consider mostly a scenario of "fast particles", whose typical Larmor radius is of the order of one tenth of the minor radius, like those considered in the earlier study [1]. Larger Larmor radii would be unrealistic in a device; employing smaller Larmor radii, conversely, non-adiabatic features are expected to be more and more difficult to detect. We will briefly return to this at the end of section 3. Notice that in higher-magnetic-fields concepts of spherical tokamaks, such as those outlined in [8], this class of particles would refer to fusion-produced alpha ions, not to beam ions.

In an axis-symmetric geometry, two exact constants of motion exist: the total energy and the momentum along the ignorable coordinate $\phi$. The existence of a third constant 
of motion ensures regularity of the motion but, conversely, the lack thereof does not necessarily imply sizeable chaotic motion. In order to confirm the existence of chaos we relied upon Poincaré maps, produced by recording particles' intersections with the plane $Z=0$ and $d Z / d t>0$. In a few cases we validated our conclusions with the explicit check of the existence of exponential separation between nearby trajectories.

We finish this section by pointing out that our simulations are collisionless, thus are meaningful as long as they do not exceed typical collision times. We estimated that the slowing-down time for a fast ion in a NSTX plasma is about $1 \mathrm{~ms}$. For comparison, our longest simulations, used for producing Poincaré maps, run up to a fraction of ms when converted into physical units, hence we feel confident that collisional effects do not alter our conclusions.

\section{Results}

\subsection{Numerical findings: a selection}

In the following figures we provide a representative selection out of our whole set of simulations. In this first set, all particles are initialized with dimensionless kinetic energy $=4.5 \times 10^{-4}$; then, the Larmor radius, averaged over the interval $0 \leq t \leq 4000$, ranges roughly between $0.75 \times 10^{-1}$ and $1.5 \times 10^{-1}$, as anticipated in the previous section. The joint conservation of the kinetic energy $K$ and of the toroidal momentum $P_{\phi}$ sets stringent constraints about the volume available to the particles. To start with, we write

$$
\begin{aligned}
& v_{\phi}=p_{\phi}-A_{\phi}(R, Z) \\
& v_{R}^{2}+v_{Z}^{2}=2 K-v_{\phi}^{2}=2 K-\left(p_{\phi}-A_{\phi}\right)^{2}
\end{aligned}
$$

For the chosen conditions, the initial $v_{\phi}$ is of order $10^{-2}$ or lesser, whereas $A_{\phi}$ may vary within a range of about $10^{-1}$ (see figure 2), and $2 K=9 \times 10^{-4}$. Hence, the right hand side of the last equation is not warranted to be positive everywhere: the particle is constrained within subregions of the whole NSTX volume. In figure (3) we show the allowed regions $(R, \mu)$ on the equatorial plane for different values of $P_{\phi}$. It is apparent how the actual allowed region is just a tiny fraction of the whole potential volume. Indeed, the range available to $\mu$ is still smaller. We rewrite, on the basis of the second

of $(9) v_{R}=w \cos (\theta), v_{Z}=w \sin (\theta), w^{2}=2 K-v_{\phi}^{2}$, and $\theta$ an appropriate angle. Through simple algebra it is possible to show that $\mu$ can be written as

$$
\mu=\frac{K}{B}-\frac{\left[B_{\phi}\left(p_{\phi}-A_{\phi}\right)+\sqrt{2 K-\left(p_{\phi}-A_{\phi}\right)^{2}}\left(B_{R} \cos (\theta)+B_{Z} \sin (\theta)\right)\right]^{2}}{2 B^{2}}(
$$

The first term, $K / B$ is just the maximum reachable $\mu$, when the whole kinetic energy is in the perpendicular component of the velocity. While, a priori, $\mu$ may vary over the whole range $(0, K / B)$, the previous equation shows that the dynamically allowed range of $\mu$ is actually that obtained by varying $\theta$ in the interval $(0,2 \pi)$.

Figure (4) summarizes our main findings about the conservation of $\mu$. It shows the pairs $(R, \mu)$ in correspondence of the intersection of the trajectory with the plane $Z=0$. 

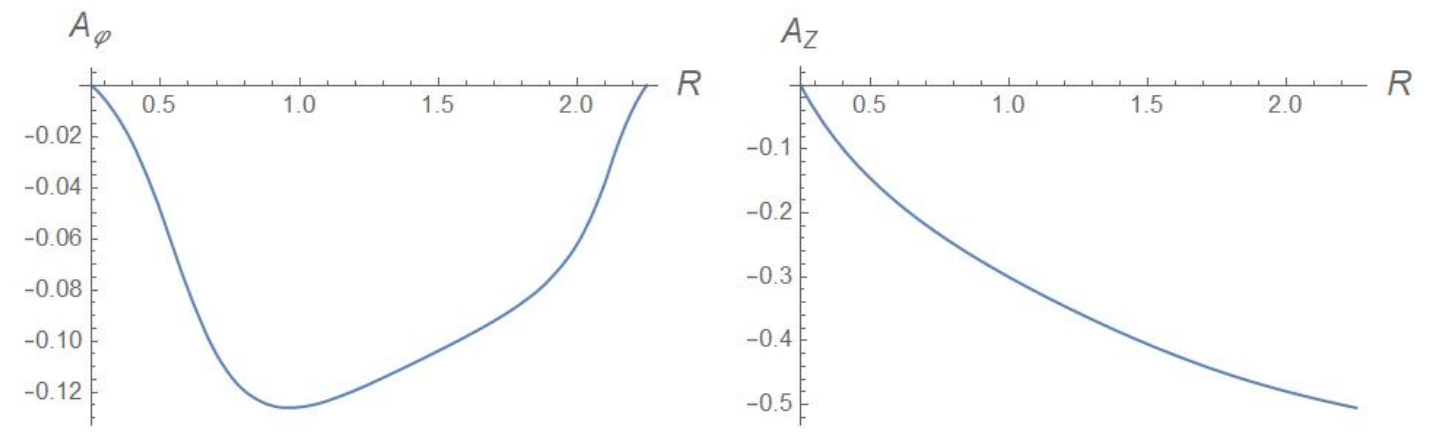

Figure 2. Left plot, radial profile along the equatorial plane $Z=0$ of the toroidal component of magnetic vector $A_{\phi}$; right plot, Z-component of the magnetic vector $A_{Z}$.

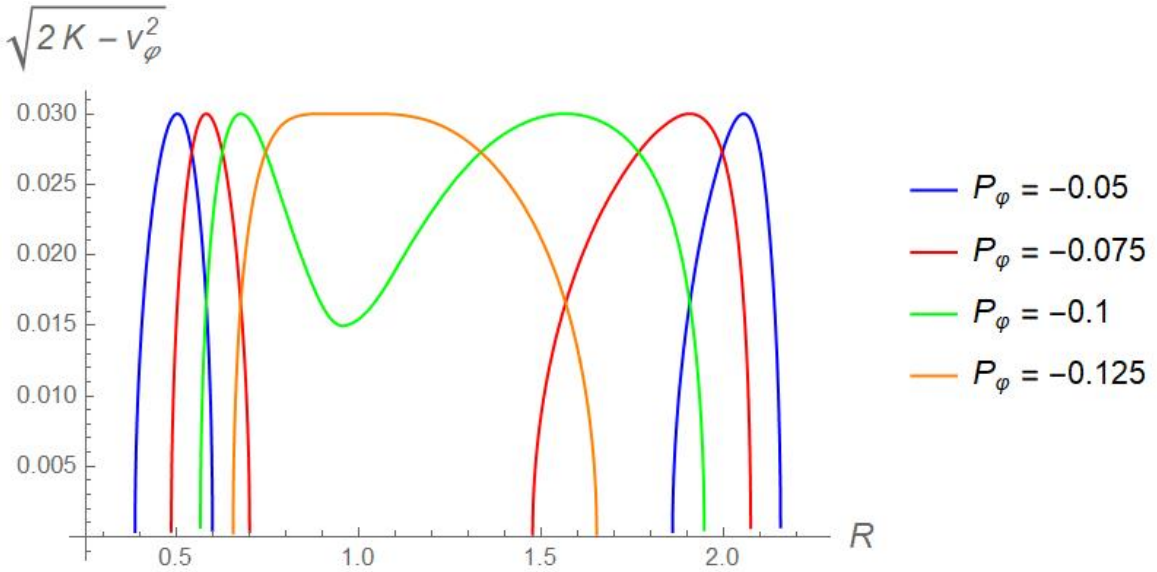

Figure 3. Radial plot along the equatorial plane $Z=0$ of the square root of Eq. (9) for different values of $P_{\phi}$. In all cases $2 K=9 \times 10^{-4}$.

Strictly speaking, they are not Poincaré sections, since these should involve phase-space coordinate as, e.g., $\left(R, P_{R}\right)$. The chosen mapping is not area-preserving, but retains the other interesting properties of Poincaré maps, i.e. KAM tori correspond to continuous curves and chaotic zones to sets of scattered points. In such a representation, if $\mu$ is conserved, each orbit appears as a series of horizontal segments. From now on, therefore, we will not distinguish between the two sections and refer to these mappings as Poincaré maps. Each row of figure (4) corresponds to a given value of $P_{\phi}$. In order to get a feeling about the scattering of the points throughout the dynamically available region of the parameters space, we shade the forbidden region, according to the previous discussion around $(9,10)$. Besides this, we plot the curves corresponding to the maximum $\mu$ reachable at each spatial position $(R, Z=0): \mu_{\max }=K / B$.

We show several orbits, each corresponding to different initial conditions, tagged by a different color. The most striking feature of these plots is the fact that a large fraction of these orbits have large fluctuations of $\mu$. In the first row, corresponding to the more external considered orbits, the relative variation of $\mu$ is between 30 and $160 \%$. In the second and third row, similar orbits are present, but the most central orbits with $\mu \leq 1$ 
have a very good conservation of $\mu$. The conservation of $P_{\phi}$ strongly limits the radial excursion of orbits, even chaotic ones. So, the strong non conservation of $\mu$ does not mean a strong loss of confinement.

We identify three sets of orbits. A first one corresponds to chaotic orbits, since their points do not match with a continuous curve, but fill a finite area. Such orbits are present for each of the three smaller values of $P_{\phi} . \mu$ is clearly not constant, since it spans a large part of the $\left[0, \mu_{\max }\right]$ domain. A second set of orbits displays a small scatter of $\mu$. They are regular, since their points define a continuous curve (orbits labelled \# 42,10,11,18). There is, finally a third type of orbit which features a significant dispersion in $\mu$, yet the overall appearance of the trajectory is regular $(\# 17,19)$. The second and third regimes represent a transition between the truly adiabatic and the super-adiabatic dynamics described by [1]: $\mu$ oscillates over a time scale substantially larger than the Larmor period, but does not drift away from a constant average value. The corresponding dynamics is still regular. Instead, the first set of orbits is a genuine example of non-adiabatic/chaotic dynamics.

\subsection{Analysis of the non-adiabatic dynamics}

We now investigate the physical causes which lead to the genuine non-adiabatic chaotic dynamics and to the failure of the guiding-center approximations. The magnetic moment is associated to the Larmor rotation around the magnetic field axis which, in a uniform magnetic field, corresponds trivially to a closed circuit. In a curved magnetic field, instead, no such circuit can be drawn any longer, but the difference in the position after a whole Larmor rotation, is smaller and smaller as particle velocity decreases. This difference is by no means small at the positions where $\mu$ changes appreciably. While performing its Larmor rotation, the particle simultaneously drifts. In a static magnetic field, the well-known gradient and curvature drifts exist:

$$
u_{\nabla B}=K_{\perp} \frac{\mathbf{B} \times \nabla B}{B^{3}}, \quad u_{\text {curv }}=2 K_{\|} \frac{\mathbf{R}_{\text {curv }} \times \mathbf{B}}{R_{\text {curv }}^{2} B^{2}}
$$

Within a Larmor period, which-in our units-is $T=2 \pi / B$, particles drift by a length which, on the basis of the structure of the previous equation, scales like $K B^{-2}$. This has to be compared with the movement due to Larmor rotation, i.e., the Larmor radius, which scales like $K^{1 / 2} B^{-1}$. Therefore, it is intuitive that the smaller the magnetic field becomes locally, the more difficult it is for the circuit to close. In order to make quantitative our statement we resort to two numerical markers. They are expected to convey roughly the same information, yet their sensitivity may be different. The first quantity is

$$
c(t)=\hat{\mu}(t) \cdot \hat{\mathbf{b}}(t)
$$

$\hat{b}$ is the unit vector of the local magnetic field; $\hat{\mu}$ is the unit vector defining the local direction of the magnetic dipole moment made by the microscopic spire produced by the rotating particle (we describe in the Appendix A, for the reader's convenience, how 

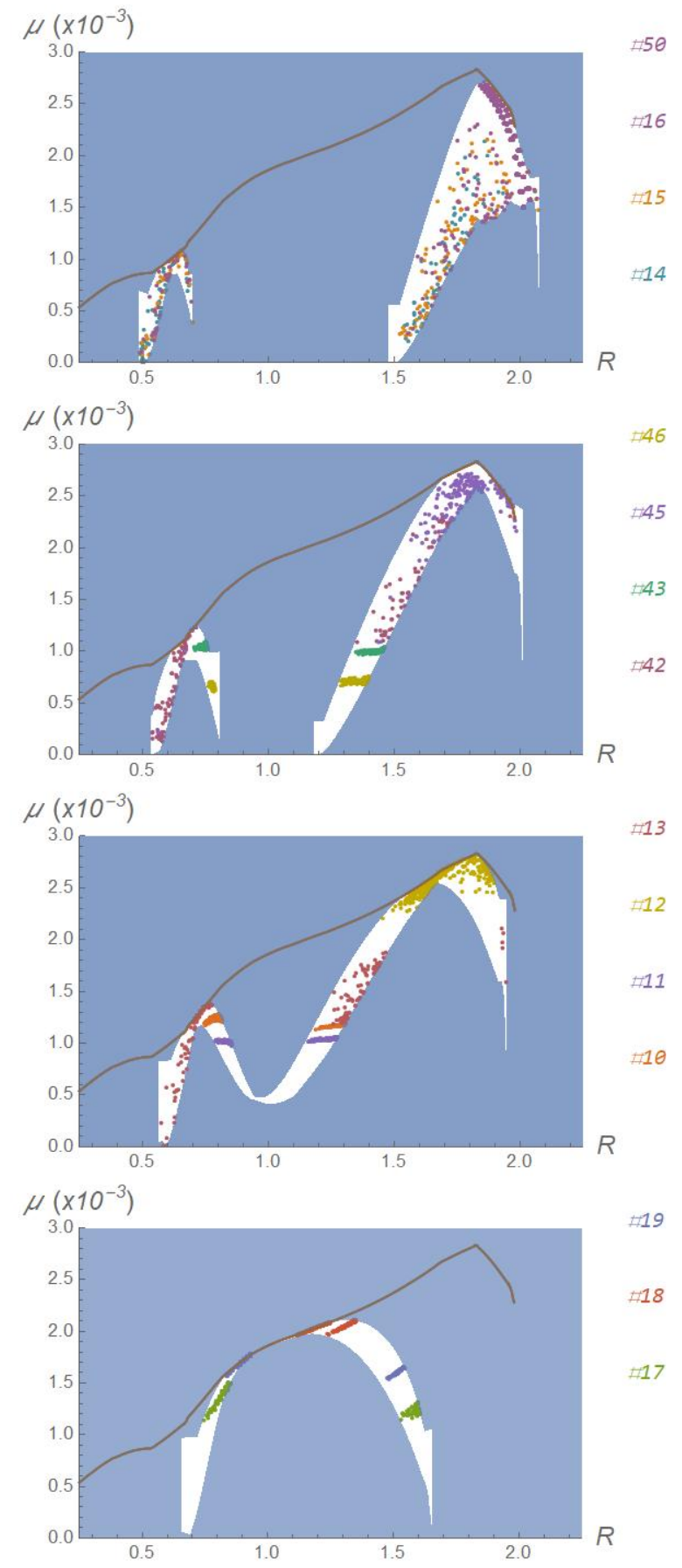

Figure 4. Each subplot includes Poincaré maps corresponding to two or more trajectories, in the plane $(R, \mu)$. Each trajectory is identified consistently throughout the whole paper by a unique color and identifier number. Plots are grouped on the basis of the toroidal momentum $P_{\phi}$ : from top to bottom $P_{\phi}=-0.075,-0.09,-0.1,-0.125$. Dashed areas mark the regions forbidden to the particle according to the discussion around $(9,10)$. 
$\hat{\mu}$ is computed from numerical data). Thus, $c$, the cosine of the angle between the two arrays, quantifies the degree of alignment of $\hat{\mu}$ with the direction of the local magnetic field. In an adiabatic regime, $\hat{\mu}$ remains aligned with $\hat{\mathbf{b}}$ at all times, and $c=1$ everywhere.

The second marker quantifies the amount of non-uniformity of the magnetic field orientation as seen by the particle during one rotation:

$$
n(t)=\hat{\mathbf{b}}(t) \cdot \hat{\mathbf{b}}(t+T), \quad T=2 \pi / B
$$

For adiabaticity to hold, $n$ must stay close to 1 . Notice that both markers are meaningful for curved magnetic fields: in straight magnetic fields, both $c(t)$ and $n(t)$ are always unitary, even though non-adiabatic dynamics may exist.

Figure 5 shows time traces for several quantities: the averaged moment $\langle\mu\rangle$ (where the fast oscillations over the Larmor time scale are removed through a moving average $\langle\mu\rangle$ done over a time window equal to the local Larmor period $2 \pi / B$, in order not to overburden the plot), the $R$ and $Z$ coordinates, the $c$ and $n$ markers. The plots refer to the same runs as figure (4), however the visualized time span is much shorter than that employed for producing the Poincaré maps: about 1/15th. In order to appreciate the time scales involved, we remind that, with the chosen units, the Larmor period is in the range $20 \div 40$ time units (see figure (1)

We enumerate the conclusions resulting from the inspection of the plots: (i) Superadiabatic dynamics corresponds to slow large oscillations of $\langle\mu\rangle$ over a mean value which remains constant in time. Conversely, genuine non-adiabatic dynamics features intervals where $\langle\mu\rangle$ is fairly constant separated by sudden jumps (i.e. occurring over time scales below or about the Larmor period) towards a fairly different value. These results are consistent with earlier studies $[1,2,9,10,11]$. (ii) Perfect alignment is seldom reached, $c<1$ almost everywhere, but $c$ has severe drops in coincidence with $\langle\mu\rangle$ jumps. (iii) The condition $n=1$ is well fulfilled everywhere except, again, in coincidence with jumps of $\langle\mu\rangle$. Thus, both $c$ and $n$ are good proxies for the breakup of the adiabaticity condition. The breakup of adiabatic dynamics is here to be understood as a consequence of an orbit crossing a region of (a) small magnetic field amplitude and (b) large variation of the orientation of the magnetic field, with (c) a large enough velocity. Conditions (a) makes large the argument $T$ in Eq. (13) and, together with conditions (b,c), entails a large difference between $\hat{b}(t)$ and $\hat{b}(t+T)$ such that in the particle frame the condition $n \approx 1$ does not hold. Equivalently, during one single Larmor rotation, the magnetic field rotates too fast for the magnetic moment to align with it.

We expect the departure from adiabatic dynamics to be smaller and smaller as the particle velocity decreases. Accordingly, we made a few exercises reducing it. Figure (6) displays a sample of the results, and shows the non-conservation of $\mu$ for particles with reduced speed, confirming our expectations. It is interesting to note that the smallest velocity appearing in the simulation is in the thermal velocity regime and still features a faint degree of non-adiabaticity. This result must not be overestimated, though: at 


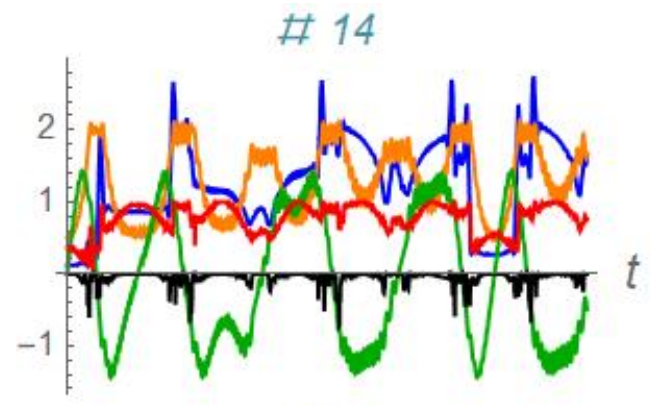

\# 16

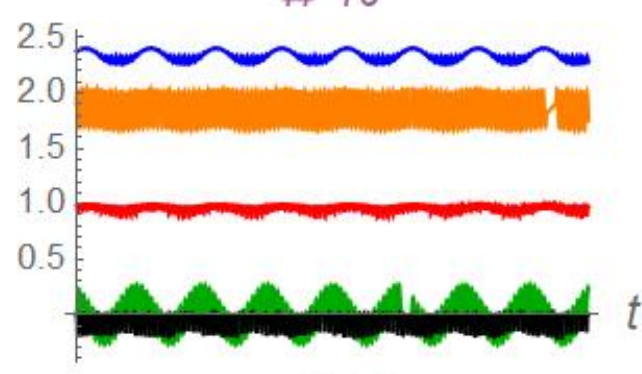

\# 13

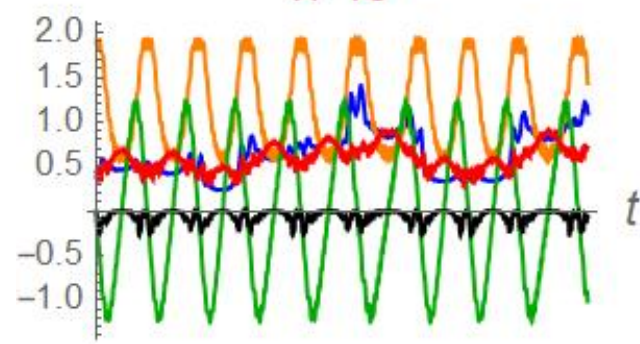

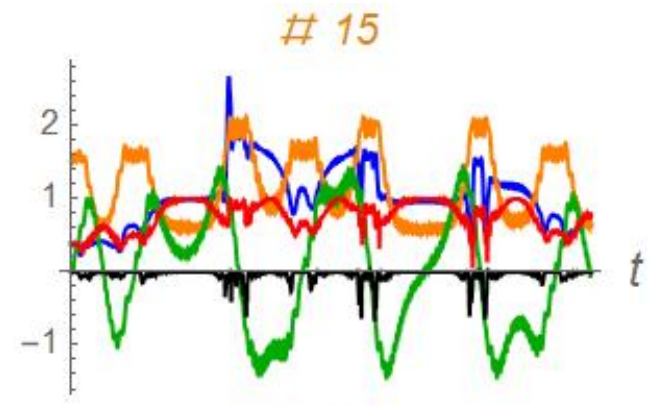

\# 42

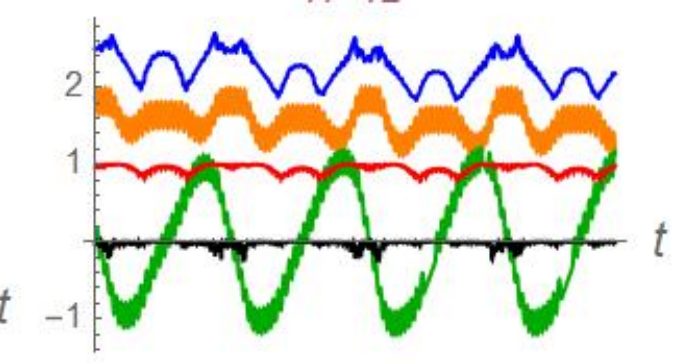

\# 17

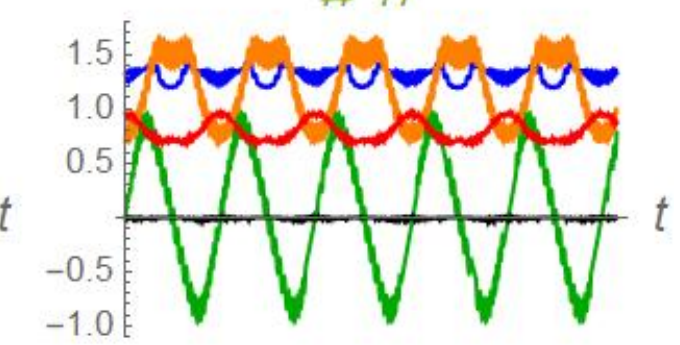

Figure 5. All plots show time traces of $\langle\mu\rangle$ (blue curve), $R$ (orange curve), $Z$ (green curve), $c$ (red curve), $n-1$ (black curve) for a few trajectories, both chaotic and regular. The time window extends up to $t=4000$ : about $1 / 15$ th of the whole simulation time used for producing Poincaré maps.

these small energies, collisional effects interfere already at these time scales.

Finally, we address the issue of the boundary between superadiabaticity and nonadiabaticity/chaos. Figure (7) shows the time traces of $\mu$ corresponding to trajectory \#42 over the whole time range used for producing its Poincaré map, splitted into four subintervals. From figure (4) one expects this orbit to be non-adiabatic, yet from figure (5) its time trace appears closer to superadiabatic. Actually, one recovers that the time trace is very close to superadiabatic over a very long interval of time, up to roughly $t=15000$ but, eventually it turns into a non-adiabatic one. Thus, the boundary between the different kind of dynamics does not look sharp, but rather fuzzy.

\section{Discussion and conclusion}

We studied the dynamics of fast beam-like particles in an axis-symmetric version of NSTX, or of alpha particles in an appropriately rescaled reactor-grade device. Our results show that the magnetic moment $\mu$ of these particles have large oscillations, and 


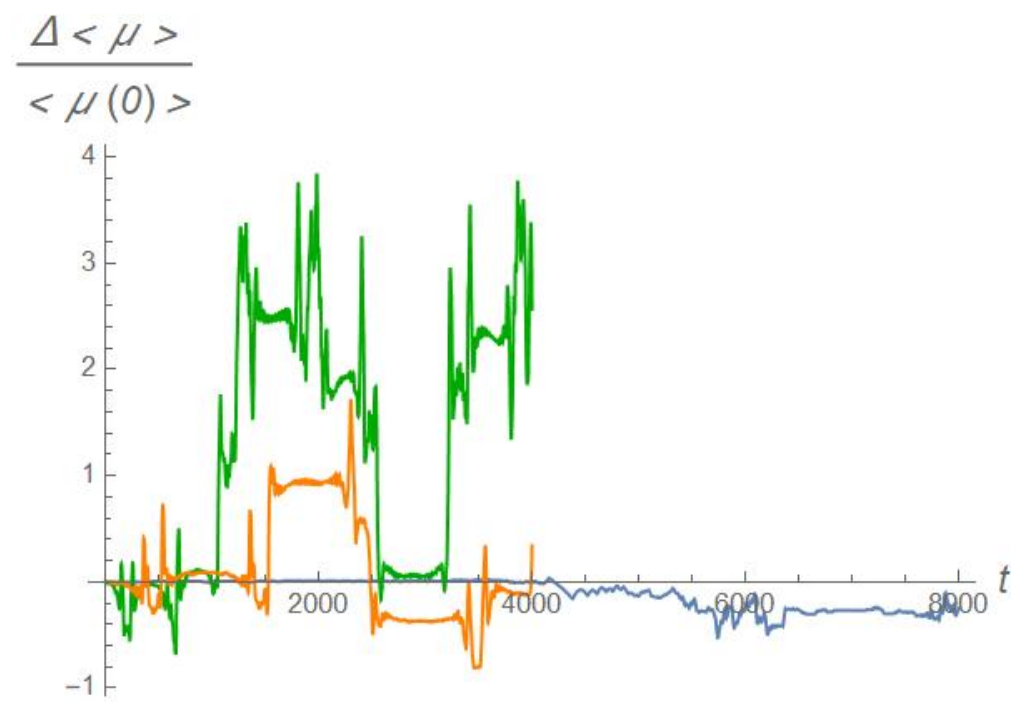

Figure 6. Time traces of the scaled $\Delta\langle\mu\rangle$ quantity. All particles are initialized at $R=0.5, Z=0$ with the same $\phi$-velocity $-6.7 \times 10^{-4}$, and thus the same momentum $P_{\phi}=-0.5$. Total kinetic energy is varied, though: $K=4.5 \times 10^{-4}$ (green curve); $K=1.2 \times 10^{-4}$ (orange curve); $K=7.25 \times 10^{-7}$ (blue curve). Because of the extremely small velocity in this latter case, the simulation has been run for twice the standard time span, in order to allow for non-adiabatic effects to appear. Actually, they start showing exactly when the previous two simulations stop.

\# 42
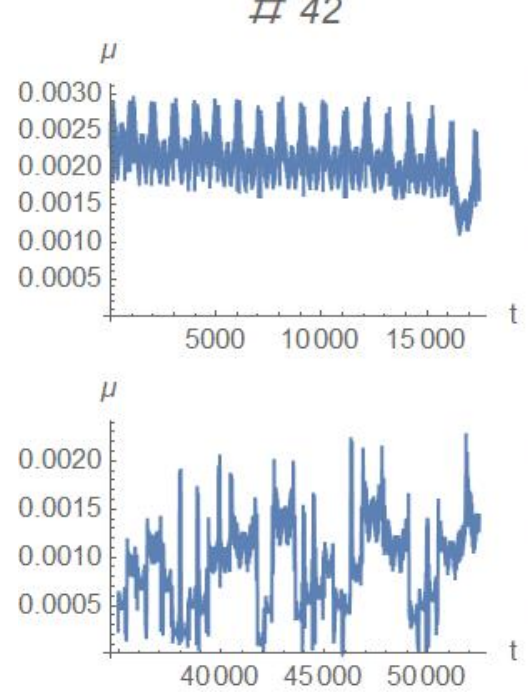
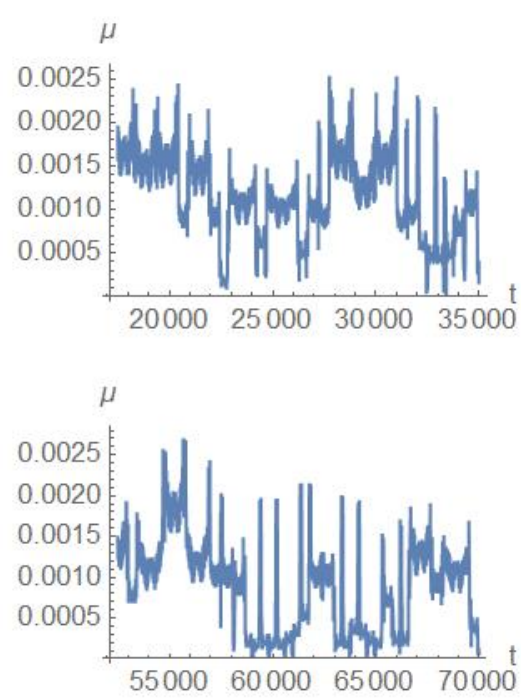

Figure 7. Time traces of $\langle\mu\rangle$ splitted into four subintervals, corresponding to orbit \#42 
even chaotic ones, in a sizable domain of the particle initial conditions. The breakup of adiabaticity occurs when an orbit with a large enough velocity crosses a region of low magnetic field amplitude with a large variation of the orientation of this field.

These large oscillations of $\mu$ have both practical and physical consequences. First, the use of guiding-center or gyrokinetic calculations for such orbits is questionable. Second, the capability of these particle to excite Alfvénic instabilities decreases, since the velocity of the particle fluctuates with respect to the phase-velocity of the Alfvén wave, which imposes a fluctuating sign to the energy exchanges with this wave. These two facts might explain a result displayed in figure 8 of [12], which shows the total neutron rate drop in NSTX versus scaled TAE amplitude: the numerical calculation performed with the guiding-center ORBIT code overestimates the experimental value by a factor about 4 . Our calculations imply that Alfvén waves are inefficient in the outer part of the confined plasma, which are good news for the confinement of fast particles.

Even when chaotic orbits are present, the conservation of the toroidal momentum $P_{\phi}$ sets strong constraints about the volume available to the particles, and their radial diffusion stays bounded. However this will no longer be the case in the presence of magnetic ripple.

\section{Appendix A}

Vector $\hat{\mu}$ may be computed using just the recorded particle trajectory $\mathbf{s}(t)$ in terms of the Serret-Frńet triad: the tangent to the trajectory $\hat{\mathbf{T}}$, its normal $\hat{\mathbf{N}}$, and the binormal $\hat{\mathbf{T}} \times \hat{\mathbf{N}}$

$$
\hat{\mathbf{T}}=\frac{d \mathbf{s}}{d t} \times \frac{1}{|d \mathbf{s} / d t|}, \quad \hat{\mathbf{N}}=\frac{d \hat{\mathbf{T}}}{d t} \times \frac{1}{|d \hat{\mathbf{T}} / d t|}
$$

and

$$
\hat{\mu}=\hat{\mathbf{T}} \times \hat{\mathbf{N}}
$$

This procedure, however, involves computing second derivatives of $\mathbf{s}(t)$ which may be very noisy for discretely sampled data. For numerical purposes, thus, we adopted the following procedure: first, the velocity of the particle is computed from the recorded trajectory

$$
\mathbf{v}=\frac{d \mathbf{s}}{d t} \equiv v \hat{\mathbf{T}}
$$

The acceleration a may be written both in terms of the derivative of $\mathbf{v}$ (recalling that $d v / d t=0)$

$$
\mathbf{a}=\frac{d \mathbf{v}}{d t}=v \frac{d \hat{\mathbf{T}}}{d t}=v \mathbf{N}^{\prime}
$$

as well as in terms of the law of motion:

$$
\mathbf{a}=\mathbf{v} \times \mathbf{B}=v B \hat{\mathbf{T}} \times \hat{\mathbf{b}}
$$


The vector $\mathbf{N}^{\prime}$ is parallel to the normal $\mathbf{N}$, but is not unitary.

By equating these two expressions we get

$$
\mathbf{N}^{\prime}=B \hat{\mathbf{T}} \times \hat{\mathbf{b}}, \quad \mathbf{N}=\mathbf{N}^{\prime} / \sqrt{\mathbf{N}^{\prime} \cdot \mathbf{N}^{\prime}}
$$

This latter recipe involves only $\mathbf{s}$ and its first derivative, and is numerically much less noisy.

[1] J. Carlsson, Phys. Plasmas 8, 4725 (2001)

[2] V.A. Yavorskij et al, Nucl. Fusion 42, 1210 (2002)

[3] D. Pfefferlé, J. P. Graves, and W. A. Cooper, Plasma Phys. Control. Fusion 57, 054017 (2015)

[4] N. N. Gorelenkov, S. D. Pinches, and K. Toi, Nucl. Fusion 54, 125001 (2014)

[5] V.A. Yavorskij, et al, Plasma Phys. Control. Fusion 43, 249 (2001).

[6] In the original paper $\alpha$ is given a negative value, which is suggested to lie within the range $-2 \leq \alpha \leq 0$. However, a negative $\alpha$ would imply a negative triangularity, i.e. a plasma pointing towards the high-field-side.

[7] R.I. McLachlan and P. Atela, Nonlinearity 5, 541 (1992)

[8] Y.-K. M. Peng and D.J. Strickler, Nucl. Fusion 26, 769 (1986)

[9] A. Garren et al, Proceedings of the Second UN International Conference on the Peaceful uses of Atomic Energy, paper P/383 (1958)

[10] R.J. Hastie et al, Proc. 3rd Int. Conf. on Plasma Physics and Controlled Nuclear Fusion Research, (Novosibirsk, 1968) vol 1, (Vienna, IAEA), p 389

[11] J.E. Howard, Phys. Fluids 14, 2378 (1971)

[12] E. Fredrickson et al, Nucl. Fusion 53, 013006 (2013) 